FACTA UNIVERSITATIS (NIŠ)

Ser. Math. Inform. Vol. 35, No 5 (2020), 1439-1449

https://doi.org/10.22190/FUMI2005439G

\title{
SOLITARY WAVE SOLUTIONS FOR SPACE-TIME FRACTIONAL COUPLED INTEGRABLE DISPERSIONLESS SYSTEM VIA GENERALIZED KUDRYASHOV METHOD
}

\begin{abstract}
Ahmed A. Gaber and Hijaz Ahmad
(C) 2020 by University of Niš, Serbia | Creative Commons Licence: CC BY-NC-ND

Abstract. In this article, space-time fractional coupled integrable dispersionless system has been considered, and we have used fractional derivative in the sense of modified Riemann-Liouville. The fractional system has been reduced to an ordinary by fractional transformation and the generalized Kudryashov method is applied to obtain exact solutions. We also testify performance as well as the precision of the applied method by means of numerical tests for obtaining solutions. The obtained results have been graphically presented to show the properties of the solutions.
\end{abstract}

Keywords. integrable dispersionless system; fractional derivative; differential system.

\section{Introduction}

In recent years, fractional differential equations have gained much attention from researchers due to thier numerous applications in many fields of sciences and engineering. These equations are widely used to describe various phenomena in many fields such as the fluid flow, electro chemistry, scattering theory, transport theory, probability, elasticity, control theory, potential theory, signal processing, image processing, diffusion theory, kinetic theory, systems identification, biology and other areas $[1,2]$. The first application of fractional calculus was introduced by Abel [3] in the solution of an integral equation that was arisen in the formulation of the tautochronous problem. This problem deals with the determination of the shape of a frictionless plane curve through the origin in a vertical plane along with a particle of mass $\mathrm{m}$ can fall in a time that is independent of the starting position [4].

Travelling wave methods have an important role to obtain solutions that are described and explained these natural phenomena. Most famous of by these effective methods are $\left(\mathrm{G}^{\prime} / \mathrm{G}\right)$-expansion method $[5-6]$, variational iteration algorithm-I

Received July 08, 2020; accepted November 07, 2020

2020 Mathematics Subject Classification. Primary 26A33; Secondary 34K37 
[7 - 8], Exp-function method [9], fractional iteration algorithm [10 - 11], Generalization of He's Exp-Function Method [12], reproducing Kernal method [13], a new extended Auxiliary equation method [14], variational iteration algorithm-II $[15-16]$ and Modified Kudryashov method [17 - 19]. In this paper, we use generalized Kudryashov method for finding the exact solutions of space-time fractional coupled integrable dispersionless system.

\section{Properties of fractional derivatives}

In this paper, we consider the most common definition named in modified RiemannLiouville derivative which is defined [20 - 26]

$$
D_{t}^{\gamma} u(t)=\left\{\begin{array}{cc}
\frac{1}{\Gamma(-\gamma)} \int_{0}^{t}(t-\tau)^{-\gamma-1}(u(\tau)-u(t)) d \tau & , \gamma<0, \\
\frac{1}{\Gamma(-\gamma)} \frac{d}{d t} \int_{0}^{t}(t-\tau)^{-\gamma-1}(u(\tau)-u(t)) d \tau, & 0<\gamma \leq 1, \\
\left(u^{(n-1)}(\tau)\right)^{(\gamma-n-1)}, & n-1<\gamma \leq n, n \geq 2
\end{array}\right.
$$

where $u: R \rightarrow R, t \rightarrow u(t)$, denotes a continuous function.

Property 1 ,

$$
D_{t}^{\gamma} t^{r}=\frac{\Gamma(1+r)}{\Gamma(1+r-\gamma)} t^{r-\gamma}, r>0
$$

Property 2,

$$
D_{t}^{\gamma}(u(t) g(t))=g(t) D_{t}^{\gamma} u(t)+u(t) D_{t}^{\gamma} g(t)
$$

Property 3,

$$
D_{t}^{\alpha} u(g(t))=\frac{d u(g(t))}{d g(t)} D_{t}^{\alpha} g(t)
$$

\section{Description of the method for FDEs}

Consider a given nonlinear wave equation

$$
N\left(u, D_{t}^{\alpha} u, D_{x}^{\alpha} u, D_{x}^{2 \alpha} u_{x x}, D_{t}^{2 \alpha} u, D_{t}^{\alpha} D_{x}^{\alpha} u, \ldots\right)=0,
$$

we seek its wave solutions

$$
u=U(\eta), \quad \eta=\frac{h_{i} x_{i}^{\alpha}}{\Gamma(1+\alpha)}+\frac{g t^{\alpha}}{\Gamma(1+\alpha)}, \quad i=1,2, \ldots
$$


Consequently, Eq. (5) is reduced to the ordinary differential equation (ODE) by transformation:

$$
U\left(u, g u^{\prime}, h u^{\prime}, g^{2} u^{\prime \prime}, h^{2} u^{\prime \prime}, \ldots\right)=0 .
$$

The generalized Kudryashov method (GKM) is based on the assumption that the travelling wave solutions can be expressed in the following form

$$
u(\eta)=\sum_{i=0}^{m} \frac{a_{i}}{(1+\phi(\eta))^{i}},
$$

where $m$ is positive integer which are unknown to be further determined, $a_{i}$ are unknown constants. In addition, $\phi(\eta)$ satisfies Riccati equation

$$
\phi^{\prime}(\eta)=A+B \phi(\eta)+C \phi^{2}(\eta) .
$$

We obtained a type of solutions of Eq. (9)

Family 1: $A$ and $B$ are free constants, $C \neq 0$

$$
\phi(\eta)=\frac{-B+\sqrt{4 A C-B^{2}} \tan \left(\frac{1}{2}\left(\sqrt{4 A C-B^{2}}\left(\eta+d_{0}\right)\right)\right)}{2 C} .
$$

Family 2: $A=0, B \neq 0$, and $C$ is a free constant

$$
\phi(\eta)=\frac{-B \exp \left(B \eta+B d_{0}\right)}{C \exp \left(B \eta+B d_{0}\right)-1} .
$$

Family 3: $A$ is free constant, $B \neq 0$, and $C=0$

$$
\phi(\eta)=\frac{-A}{B}+\frac{1}{B} \exp (B \eta)
$$

Family $4: A=0, B=-1$ and $\mathrm{C}=-1$

$$
\phi(\eta)=\frac{-d_{0}}{\exp (\eta)+d_{0}} .
$$

\section{Space-time fractional coupled Integrable Dispersionless system}

We consider the space-time fractional coupled Integrable Dispersionless (CID) system

$$
\begin{aligned}
\frac{\partial^{2 \alpha} u}{\partial t^{\alpha} \partial x^{\alpha}}+\frac{\partial^{\alpha}}{\partial x^{\alpha}}(v w) & =0 \\
\frac{\partial^{2 \alpha} v}{\partial t^{\alpha} \partial x^{\alpha}}-2 v \frac{\partial^{\alpha} u}{\partial x^{\alpha}} & =0
\end{aligned}
$$




$$
\frac{\partial^{2 \alpha} w}{\partial t^{\alpha} \partial x^{\alpha}}-2 w \frac{\partial^{\alpha} u}{\partial x^{\alpha}}=0
$$

where $\mathrm{u}, \mathrm{v}$ and $\mathrm{w}$ are all functions of $\mathrm{x}$ and t. Eqs. (10) describes the currentfed string within an external magnetic field $[27,28]$. This equations wase presented and solved by the inverse scattering method [29], the exp-function method [30] and residue harmonic balance [31].

We perform the transformation $\eta=\frac{h x^{\alpha}}{\Gamma(1+\alpha)}+\frac{g t^{\alpha}}{\Gamma(1+\alpha)}$, Eq. (10) can be reduced into an ODEs

$$
\begin{array}{cc}
g h U^{\prime \prime}+h\left(V W^{\prime}+W V^{\prime}\right)= & 0, \\
g h V^{\prime}-2 h V U^{\prime} & =0, \\
g h W^{\prime}-2 h W U^{\prime}= & 0
\end{array}
$$

where $U^{\prime}=\frac{\partial U}{\partial \eta}$.

We can freely know that the solution does not depend on the balancing the highest order linear and nonlinear terms [32]. For simplicity, we set $\mathrm{i}=2$, we have:

$$
\begin{aligned}
U(\eta) & =a_{0}+\frac{a_{1}}{1+\phi(\eta)}+\frac{a_{2}}{(1+\phi(\eta))^{2}} \\
V(\eta) & =b_{0}+\frac{b_{1}}{1+\phi(\eta)}+\frac{b_{2}}{(1+\phi(\eta))^{2}} \\
W(\eta) & =r_{0}+\frac{r_{1}}{1+\phi(\eta)}+\frac{r_{2}}{(1+\phi(\eta))^{2}}
\end{aligned}
$$

Substituting Eq. (12) into Eq. (11), equating to zero the coefficients of all powers of $\phi(\eta)$ yields a set of algebraic equations for $\mathrm{a}_{i}, \mathrm{~b}_{i}, \mathrm{r}_{i}$.

$$
\begin{aligned}
& h\left(6 B C r_{0} a_{1}+2 A r_{0} a_{1}+3 g r_{1} A B+4 B r_{0} a_{2}+g r_{1} B^{2} C+2 B C r_{1} a_{1}+4 g r_{2} B^{2}\right)=0, \\
& h\left(2 A b_{0} a_{1}+6 B b_{0} a_{1}+4 g b_{2} B^{2}+3 g b_{1} A B+g b_{1} B^{2}+4 B C b_{0} a_{2}+2 B b_{1} a_{1}\right)=0, \\
& h\left(2 B r_{0} a_{1}+g r_{1} B^{2}\right)=0, h\left(g b_{1} B^{2}+2 B b_{0} a_{1}\right)=0, h\left(g a_{1} B^{2}-B b_{0} r_{1}-B b_{1} r_{0}\right)=0, \\
& h\left(g a_{1} B^{2}-A b_{1} r_{0}+3 g a_{1} A B-2 B C b_{0} r_{2}-A b r_{1}-2 B b_{2} r-2 B b_{1} r_{1}-3 B C b_{0} r_{1}\right. \\
& \left.+4 g a_{2} B^{2}-3 B b_{1} r_{0}\right)=0, \\
& h\left(2 g r_{1} A^{2}+6 g r_{2} A^{2}+2 A r_{0} a_{1}+4 A r_{0} a_{2}+2 A r_{1} C a_{1}+4 A r_{1} a_{2}+2 A C r_{2} a_{1}\right. \\
& \left.+4 A r_{2} a_{2}-2 g r_{2} A B-g r_{1} A B\right)=0 \\
& h\left(4 A b_{1} a_{2}+2 A b_{2} a_{1}+4 A b_{2} a_{2}+2 A b_{0} a_{1}+4 A b_{0} a_{2}+6 g b_{2} A^{2}-g b_{1} A B\right. \\
& \left.-2 g b_{2} A B+2 A b_{1} a_{1}+2 g b_{1} A^{2}\right)=0 \\
& h\left(-2 A b_{1} r_{1}+2 g a_{1} A^{2}+6 g a_{2} A^{2}-A C b_{1} r_{0}-4 A b_{2} r_{2}-3 A C b_{1} r_{2}-2 A b_{2} r_{0}\right.
\end{aligned}
$$




$$
\left.-3 A b_{2} r_{1}-A b_{0} r_{1}-g a_{1} A B-2 A b_{0} r_{2}-2 g a_{2} A B\right)=0,
$$

Solving the system of algebraic equations with the help of Maple, we obtain the solutions organized in the following cases:

Case (1)

$$
\begin{aligned}
& a_{1}=-g A-g C+g B, b_{0}=\frac{-1}{4 r_{0}} g^{2}\left(4 C^{2}-4 B C+B^{2}\right), \\
& b_{1}=\frac{-1}{2 r_{0}} g^{2}\left(-2 A C-2 C^{2}+3 B C+A B-B^{2}\right), \\
& r_{1}=\frac{2 r_{0}(A+C-B)}{-2 C+B}, a_{0} \text { is arbitrary, } b_{2}=r_{2}=a_{2}=0 .
\end{aligned}
$$

Substituting these results into (11) and with the aid of families 1-4, we obtain the following multiple soliton-like and periodic solutions for space-time fractional CID system

$$
\begin{aligned}
& u(x, t)=a_{0}+\frac{-g A-g C+g B}{1+\frac{-B+\sqrt{4 A C-B^{2}} \tan \left(\frac{1}{2}\left(\sqrt{4 A C-B^{2}}\left(\eta+d_{0}\right)\right)\right)}{2 C}}, \\
& v(x, t)=\frac{-1}{4 r_{0}} g^{2}\left(4 C^{2}-4 B C+B^{2}\right)+\frac{-2 C g^{2}\left(-2 A C-2 C^{2}+3 B C+A B-B^{2}\right)}{2 r_{0}\left(2 C-B+\sqrt{4 A C-B^{2}} \tan \left(\frac{1}{2}\left(\sqrt{4 A C-B^{2}}\left(\eta+d_{0}\right)\right)\right)\right)}, \\
& w(x, t)=r_{0}+\frac{4 C r_{0}(A+C-B)}{(-2 C+B)\left(2 C+-B+\sqrt{4 A C-B^{2}} \tan \left(\frac{1}{2}\left(\sqrt{4 A C-B^{2}}\left(\eta+d_{0}\right)\right)\right)\right)},
\end{aligned}
$$

where $\eta=\frac{h x^{\alpha}}{\Gamma(1+\alpha)}+\frac{g t^{\alpha}}{\Gamma(1+\alpha)}$.

\section{Case (2)}

$$
\begin{gathered}
a_{1}=g C, b_{1}=\frac{g^{2} C^{2}}{r_{1}}, B=2 C, r_{1} \text { is arbitrary } \\
r_{0}=b_{0}=a_{2}=b_{2}=r_{2}=A=0 .
\end{gathered}
$$

Substituting these results into (11) and with the aid of families 1-4, we obtain the following multiple soliton-like and periodic solutions for space-time fractional CID system

$$
\begin{aligned}
& u(x, t)=a_{0}-\frac{g C\left(C \exp \left(B\left(\frac{h x^{\alpha}}{\Gamma(1+\alpha)}+\frac{g t^{\alpha}}{\Gamma(1+\alpha)}\right)+B d_{0}\right)-1\right)}{C \exp \left(B\left(\frac{h x^{\alpha}}{\Gamma(1+\alpha)}+\frac{g t^{\alpha}}{\Gamma(1+\alpha)}\right)+B d_{0}\right)+1} \\
& v(x, t)=b_{0}-\frac{g^{2} C^{2}\left(C \exp \left(B\left(\frac{h x^{\alpha}}{\Gamma(1+\alpha)}+\frac{g t^{\alpha}}{\Gamma(1+\alpha)}\right)+B d_{0}\right)-1\right)}{r_{1}\left(C \exp \left(B\left(\frac{h x^{\alpha}}{\Gamma(1+\alpha)}+\frac{g t^{\alpha}}{\Gamma(1+\alpha)}\right)+B d_{0}\right)+1\right)},
\end{aligned}
$$




$$
w(x, t)=r_{0}-\frac{r 1\left(C \exp \left(B\left(\frac{h x^{\alpha}}{\Gamma(1+\alpha)}+\frac{g t^{\alpha}}{\Gamma(1+\alpha)}\right)+B d_{0}\right)-1\right)}{C \exp \left(B\left(\frac{h x^{\alpha}}{\Gamma(1+\alpha)}+\frac{g t^{\alpha}}{\Gamma(1+\alpha)}\right)+B d_{0}\right)+1} .
$$

\section{Case (3)}

$$
\begin{gathered}
a_{1}=g(B-A), b_{0}=\frac{-g^{2} B^{2}}{4 r_{0}}, r_{1}=\frac{-2 r_{0}(A-B)}{B}, a_{0} \text { is arbitrary } \\
b_{1}=\frac{-g^{2} B(A-B)}{2 r_{0}}, a_{2}=b_{2}=r_{2}=0
\end{gathered}
$$

Substituting these results into (11) and with the aid of families 1-4, we obtain the following multiple soliton-like and periodic solutions for space-time fractional CID system

$$
\begin{gathered}
u(x, t)=a_{0}+\frac{g(B-A)}{1-\frac{A}{B}+\frac{1}{B} \exp \left(B\left(\frac{h x^{\alpha}}{\Gamma(1+\alpha)}+\frac{g t^{\alpha}}{\Gamma(1+\alpha)}\right)\right)}, \\
v(x, t)=\frac{-g^{2} B^{2}}{4 r_{0}}-\frac{g^{2} B(A-B)}{2 r_{0}\left(1-\frac{A}{B}+\frac{1}{B} \exp \left(B\left(\frac{h x^{\alpha}}{\Gamma(1+\alpha)}+\frac{g t^{\alpha}}{\Gamma(1+\alpha)}\right)\right)\right.}, \\
w(x, t)=r_{0}-\frac{-2 r_{0}(A-B)}{B\left(1-\frac{A}{B}+\frac{1}{B} \exp \left(B\left(\frac{h x^{\alpha}}{\Gamma(1+\alpha)}+\frac{g t^{\alpha}}{\Gamma(1+\alpha)}\right)\right)\right.} .
\end{gathered}
$$

\section{Discussion}

In this section, we discuss the physical explanations of the obtained solutions. Note that, the plots of the solutions (15), (17), and (19) are presented in figures 1 to 6 at specific values of the free constants. It appears that the solutions of (15), (17) and (19) depend on the sign of the magnitude $4 A C-B^{2}$. In the case of $4 A C-B^{2}>0$, the solution (15) is expressed in terms of the trigonometric tan function and hence an anti-kink wave is produced as shown by Fig. 7.1(a). Similarly the solution (19) in Fig. 7.3(a). On the other side, $4 \mathrm{AC}-\mathrm{B}^{2}<0$, the solution (17) can be expressed in terms of the hyperbolic tan function and accordingly a kink wave is resulted as displayed in Fig. 7.2(b).

On the other hand, the solutions are affected by the fractional derivatives $\alpha$, in Fig. 7.1(b) the anti-kink wave increases with increasing of $\alpha$ but the reverse effect is observed a little near off the plate $(x>0.5)$. Similarly the solution (19) in Fig. 7.1(b). Finally, Fig. 7.2(b) describes the u-solution in (17), the kink wave increases with increasing of $\alpha$ at $0<x \leq 1$ and then it stabilizes with different value of $\alpha$ at $x>1$. Accordingly, this method is capable of producing a different types of wave solutions for partial differential equations 


\section{Conclusion}

In the present paper, GKM has been successfully employed to obtain the exact solution of space-time fractional coupled integrable dispersionless system. New travelling wave technique is applied to search for the exact solitary solutions. The main advantage of the proposed method over the others is the fact that it can be applied to a wide class of nonlinear evolution equations. The modified Kudryashov [16] is special case of this technique (family 3 , take $A=0$ and $B=1$ ). Finally, the obtained results have been graphically presented to show the properties of the obtained solutions.

\section{Acknowledgments}

The authors would like to thank the deanship of scientific research of Majmaah university for the financial grant received for conducting this research

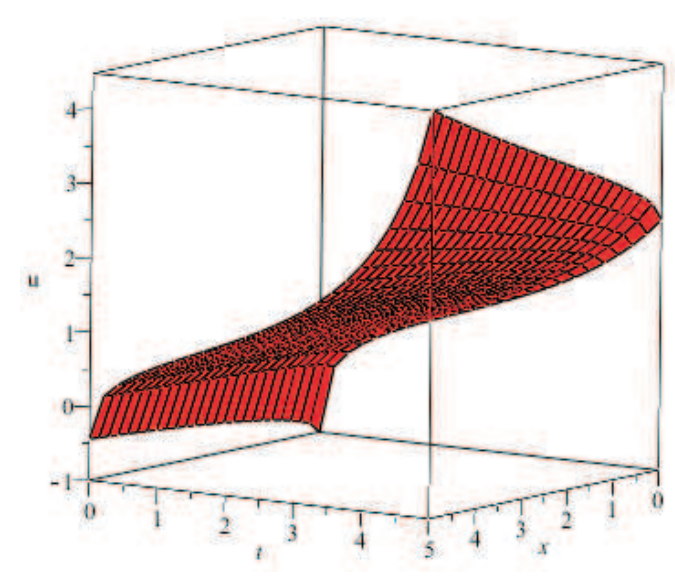

(a)

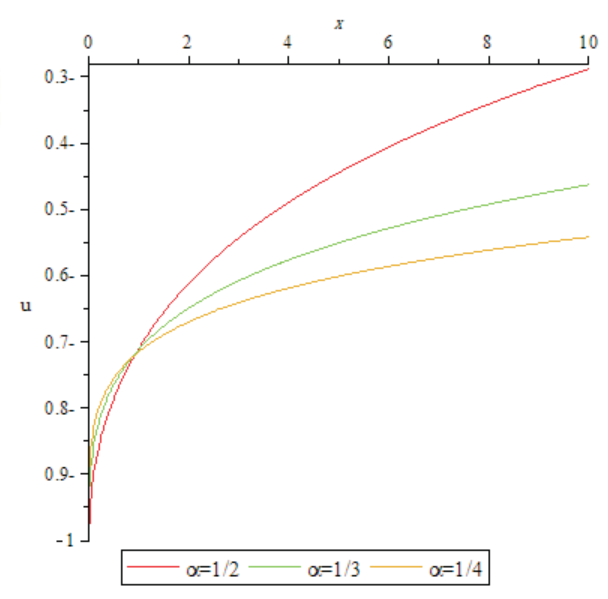

(b)

FIG. 7.1: (a) Anti-Kink wave solution of Eq. (17) where $a_{0}=a_{1}=g=A=B=C$ $=1, d_{0}=0, h=0.1$ and $\alpha=\frac{1}{2}$

(b) Anti-Kink wave solution of Eq. (17) where t $=0, a_{0}=a_{1}=g=A=B=C=1, d_{0}=0$ and $h=0.1$ 


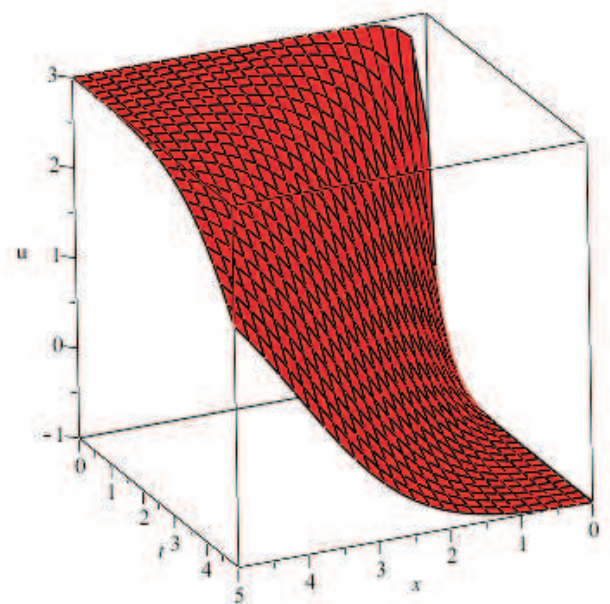

(a)

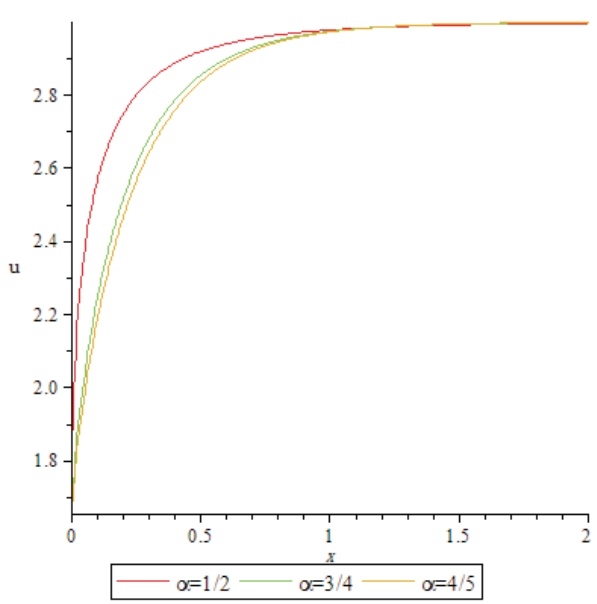

(b)

FIG. 7.2: (a) Kink wave solution of Eq. (15) where $a_{0}=1, C=2, g=-1, d_{0}=0, h=1$ and $\alpha=\frac{3}{4}$.

(b) Kink wave solution of Eq. (15) where t=0, $a_{0}=1, C=1, g=-1, d_{0}=0$ and $h=1$.

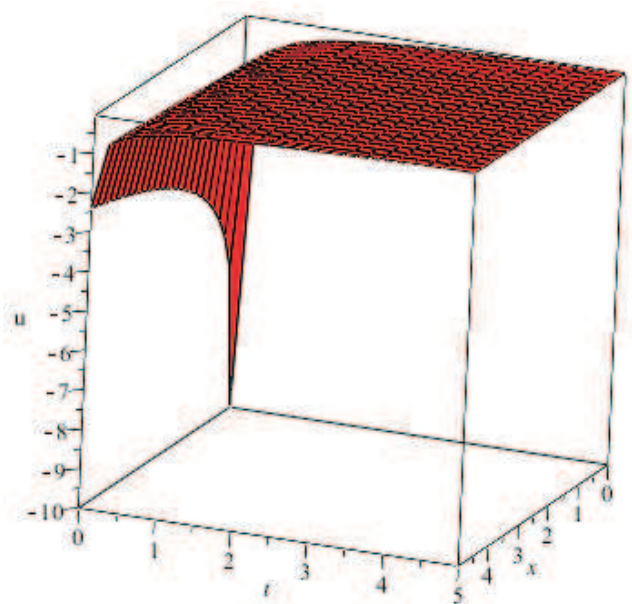

(a)

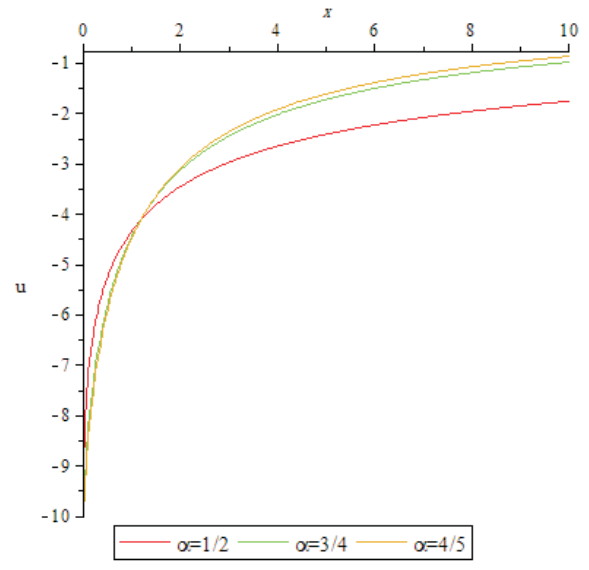

(b)

FIG. 7.3: (a) (5) Anti-Kink wave solution of Eq. (19) where $a_{0}=\mathrm{r}_{0}=1, b_{1}=-0.1, g=$ $B=1, h=0.1$ and $\alpha=\frac{1}{2}$.

(b) Anti-Kink wave solution of Eq. (19) where $a_{0}=\mathrm{r}_{0}=1, b_{1}=-0.1, g=B=1, h=0.1$ 


\section{REF E R E N C ES}

1. Zheng Bin, $\left(\mathrm{G}^{\prime} / \mathrm{G}\right)$-Expansion Method for Solving Fractional Partial Differential Equations in the Theory of Mathematical Physics, Commun. Theor. Phys. 58 (2012) 623630.

2. Mohd. Farman Ali, Manoj Sharma, Renu Jain, An Application of Fractional Calculus in Electrical Engineering, Adv. Eng. Tec. Appl. 5 (2016), 41-45.

3. N. H. Abel, Solution De Quelques Problems a L'aide D'integrals Definites. Oeuvres Completes, Grondahl Christiania, Norway,1 (1881),16-18.

4. Mehdi Dalir, Majid Bashour, Applications of Fractional Calculus, App. Math. Sci., (4) (2010), 1021 - 1032.

5. Yokus A, Durur H, Ahmad H. Hyperbolic type solutions for the couple BoitiLeon-Pempinelli system. Facta Universitatis, Series: Mathematics and Informatics. 2020;35(2):523-31.

6. Yokus A, Durur H, Ahmad H, Yao SW. Construction of Different Types Analytic Solutions for the Zhiber-Shabat Equation. Mathematics. 2020;8(6):908.

7. Ahmad H, Seadawy AR, Khan TA, Thounthong P. Analytic approximate solutions for some nonlinear Parabolic dynamical wave equations. Journal of Taibah University for Science. 2020;14(1):346-58.

8. Ahmad H, Khan TA, Stanimirovic PS, Ahmad I. Modified Variational Iteration Technique for the Numerical Solution of Fifth Order KdV Type Equations. Journal of Applied and Computational Mechanics. 2020;6(SI):1220-1227

9. Kamran Ayub, M. Yaqub Khan, Qazi Mahmood-Ul-Hassan, Solitary and periodic wave solutions of Calogero-Bogoyavlenskii-Schiff equation via exp-function methods, Computers \& Math. App., 74 (2017), 3231-3241.

10. Ahmad, H., Khan, T. A., Ahmad, I., Stanimirović, P. S., Chu, Y.-M., A new analyzing technique for nonlinear time fractional Cauchy reaction-diffusion model equations, Results in Physics, 2020, 103462. doi: https://doi.org/10.1016/j.rinp.2020.103462.

11. Ahmad, H., Akgül, A., Khan, T. A., Stanimirović, P. S., Chu, Y.-M., New Perspective on the Conventional Solutions of the Nonlinear Time-Fractional Partial Differential Equations, Complexity, 2020,2020, 8829017. doi:10.1155/2020/8829017.

12. A. A. Gaber, Symmetry analysis and Solitary wave solutions of nonlinear ion-acoustic waves equation. Int. J. Ana. App. 18 (2020) 448-460.

13. Akgül A. and Ahmad H., Reproducing Kernel Method for Fangzhuâ $€^{\mathrm{TM}_{\mathrm{S}}}$ Oscillator for Water Collection from Air, Mathematical Methods in the Applied Sciences. 2020, DOI:10.1002/mma.6853

14. Arzu Akbulut, Melike Kaplan, Auxiliary equation method for time-fractional differential equations with conformable derivative, Comput. \& Math. with Appl., 75 (2018), 876-882.

15. Ahmad, H., Khan, T. A., Stanimirović, P. S., Chu, Y.-M., Ahmad, I., Modified Variational Iteration Algorithm-II: Convergence and Applications to Diffusion Models, Complexity, 2020, 8841718. doi:10.1155/2020/8841718.

16. Ahmad H, Seadawy AR, Khan TA. Study on numerical solution of dispersive water wave phenomena by using a reliable modification of variational iteration algorithm. Mathematics and Computers in Simulation. 2020;177:13-23 
17. A. A. Gaber and et., The generalized version of Kudryashov method for nonlinear space-time fractional partial differential equations of Burgers type, Nonlinear Dynamics, Nonlinear Dyn. 95 (2019), 361-368.

18. K. Hosseini, A. Bekir, R. Ansari, New exact solutions of the conformable timefractional Cahn-Allen and Cahn-Hilliard equations using the modified Kudryashov method, Optik,132 (2017), 203-209.

19. Dipankar Kumar, Aly R. Seadawy, Atish Kumar Joardar, Modified Kudryashov method via new exact solutions for some conformable fractional differential equations arising in mathematical biology, Chinese Journal of Physics, 56 (2018), 75-85.

20. Abdelhalim Ebaid, Doaa M. M. ElSayed, Fractional Calculus Model for Damped Mathieu Equation Approximate Analytical Solution, App. Math. Sci., 6 (2012), 4075 - 4080.

21. Amit Prakash, Manoj Kumar, Kapil K. Sharma, Numerical method for solving fractional coupled Burgers equations, App. Math. Comp. 260 (2015) 314-320

22. R. Sahadevan, P. Prakash, On Lie symmetry analysis and invariant subspace methods of coupled time fractional partial differential equations, Chaos, Solitons \&Fractals 104 (2017) 107-120.

23. Qing Huang, Renat Zhdanov, Symmetries and exact solutions of the time fractional Harry-Dym equation with Riemann-Liouville derivative, Phy. A 409 (2014) 110-118.

24. Ozkan Guner, Esin Aksoy, Ahmet Bekir, Adem C. Cevikel,Different methods for (3 + 1)-dimensional space-time fractional modified KdV-Zakharov-Kuznetsov equation, Comp. \& Math. with Applications, 71(2016), 1259-1269.

25. I. Naeem, M.D. Khan, Symmetry classification of time-fractional diffusion equation, Commun Nonlinear Sci Numer Simulat 42 (2017) 560-570.

26. S. Sahoo, S. Saha Ray, Invariant analysis with conservation laws for the time fractional Drinfeld-Sokolov-Satsuma-Hirota equations, Chaos, Solitons and Fractals 104 (2017) 725-733.

27. H. Kakuhata and K. Konno, Loop Soliton Solutions of String Interacting with External Field, J. Phys. Soc. Jpn. 68 (1999) 757.

28. WANG Pan, TIAN Bo, LIU Wen-Jun, QU Qi-Xing, and JIANG Yan, Conservation Laws and Analytic Soliton Solutions for Coupled Integrable Dispersionless Equations with Symbolic Computation.

29. K. Konno and H. Oono, New Coupled Integrable Dispersionless Equations, J. Phys. Soc. Jpn. 63 (1994) 377.

30. DAI Chao-Qing, YANG Qin, and WANG Yue-Yue, New Exact Solutions of $(1+$ 1)-Dimensional Coupled Integrable Dispersionless System, Commun. Theor. Phys. 55 (2011) 622-628.

31. A.Y.T. Leung, H.X. Yang, Z.J. Guo, Periodic wave solutions of coupled integrable dispersionless equations by residue harmonic balance, Commun Nonlinear Sci Numer Simulat 17 (2012) 4508-4514.

32. A. Ebaid, An improvement on the Exp-function method when balancing the highest order linear and nonlinear terms, J. Math. Anal. Appl. 392 (2012) 1-5. 
Ahmed A. Gaber

College of Science and Humanities at Howtat Sudair

Department of Mathematics, Majmaah University,

Majmaah 11952, Saudi Arabia.

Faculty of Education, Department of Mathematics,

Ain Shams University, Heliopolis, Roxy, Egypt.

a.gaber@mu.edu.sa and aagaber6@gmail.com

Hijaz Ahmad

Section of Mathematics, International Telematic University Uninettuno,

Corso Vittorio Emanuele II, 39, 00186 Roma, Italy.

Department of Basic Sciences, University of Engineering and Technology

Peshawar, 25000, Pakistan

hijaz555@gmail.com 